\title{
Education and Outreach for the Pierre Auger Observatory
}

\author{
Gregory Snow $^{* a}$ for the Pierre Auger Collaboration ${ }^{b}$ \\ ${ }^{a}$ University of Nebraska-Lincoln \\ ${ }^{b}$ Observatorio Pierre Auger, Av. San Martín Norte 304, 5613 Malargüe, Argentina \\ E-mail: gsnow1@unl.edu \\ Full author list: http://www.auger.org/archive/authors_2016_08.html
}

\begin{abstract}
The scale and scope of the physics studied at the Pierre Auger Observatory continue to offer significant opportunities for original outreach work. Education, outreach and public relations of the Auger collaboration are coordinated in a dedicated task whose goals are to encourage and support a wide range of efforts that link schools and the public with the Auger scientists and the science of cosmic rays, particle physics, and associated technologies. We focus on the impact of the Collaboration in Mendoza Province, Argentina, and beyond. The newly renovated Auger Visitor Center in Malargüe has hosted over 105,000 visitors since 2001, and a sixth collaboration-sponsored science fair was held on the Observatory campus in November 2016. Numerous online resources, video documentaries, and animations of extensive air showers have been created for wide public release. This includes a data set of recorded air shower events which students and teachers can analyze with user-friendly online tools. Increasingly, collaborators draw on these resources to develop Auger related displays and outreach events at their institutions and in public settings to disseminate the science and successes of the Observatory worldwide. We will also highlight education and outreach activities associated with the planned upgrade of the Observatory's detector systems and future physics goals.
\end{abstract}

38th International Conference on High Energy Physics

3-10 August 2016

Chicago, USA

${ }^{*}$ Speaker. 


\section{Introduction}

Education and public outreach (EPO) have been an integral part of the Pierre Auger Observatory since its inception. The collaboration's EPO activities are organized in a separate Education and Outreach Task that was established in 1997. With the Observatory headquarters located in the remote city of Malargüe, population 28,000, early outreach activities, which included public talks, visits to schools, and courses for science teachers and students, were aimed at familiarizing the local population with the science of the Observatory and the presence of the large collaboration of international scientists in the isolated communities and countryside of Mendoza Province. As an example of the Observatory's integration into local traditions, the collaboration has participated in the annual Malargüe Day parade since 2001 with collaborators walking behind a large Auger banner (see Fig. 1). Close contact with the community fosters a sense of ownership and of being a part of the Observatory's scientific mission. The Observatory's EPO efforts have been documented in proceedings contributions to International Cosmic Ray Conferences for over a decade (see, for example, [1]). Highlights of recent outreach activities are reported here.
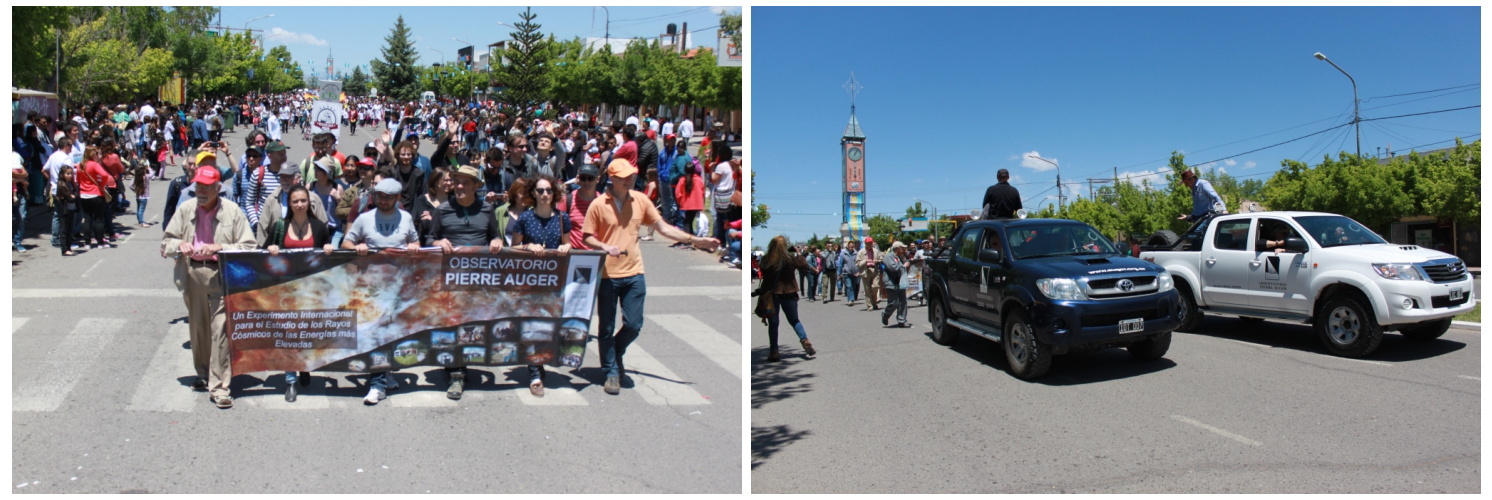

Figure 1: Left: Auger collaborators participating in the November 2016 Malargüe Day parade. Right: Observatory trucks, used for field operations, in the parade.

\section{The Auger Visitor Center in Malargüe}

The Auger Visitor Center (VC), located in the central office complex in Malargüe, continues to be a popular attraction. Through Nov. 12, 2016, the VC has hosted 105,772 visitors. Fig. 2 (left panel) shows the number of visitors since November 2001. The noticeable increase of visitors in 2009 - 2010 occurred after the opening of a nearby planetarium [2] in August of 2008. A gradual decrease in the visitor count was then observed from 2011 - 2015. However, the visitor count showed a renewed increase during the first 10.5 months of 2016, indicating the count is on track to reach over 10,000 visitors by year's end. This is attributed to a major renovation of the VC in Nov. 2015 as well as a change in the way visitors are hosted. The VC renovation transformed the space to a more engaging environment with several interactive, hands-on displays. Fig. 3 shows two such displays - an interactive Geiger counter display which allows visitors to count cosmic-ray hits during their visit in real time and two "pods" in which visitors can listen to an informative program about the Observatory. The latter are specially designed to enhance the visitor experience 
for vision-impaired visitors. In addition, visitors are now encouraged to take a self-guided tour of several attractions inside and outside the VC any time when the Observatory is staffed, in contrast to the previous mode of offering visitor presentations at prescribed times during the day. A sign at the entrance of the Observatory campus (see Fig. 2, right panel) invites visitors to the self-guided tour attractions. The renovated VC can be easily reconfigured so that large school groups can be seated and enjoy a presentation by the Observatory staff.

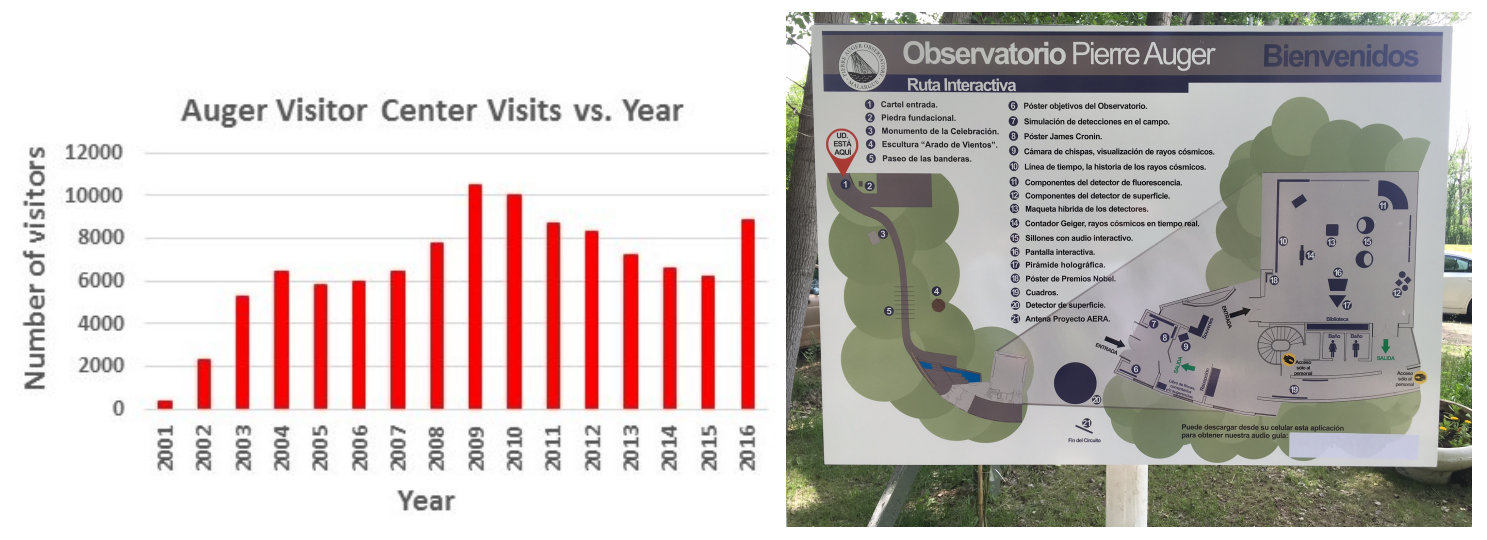

Figure 2: Left: Auger Visitor Center visits vs. year. Right: Sign at the entrance of the Observatory campus illustrating self-guided tour attractions inside and outside the Visitor Center.
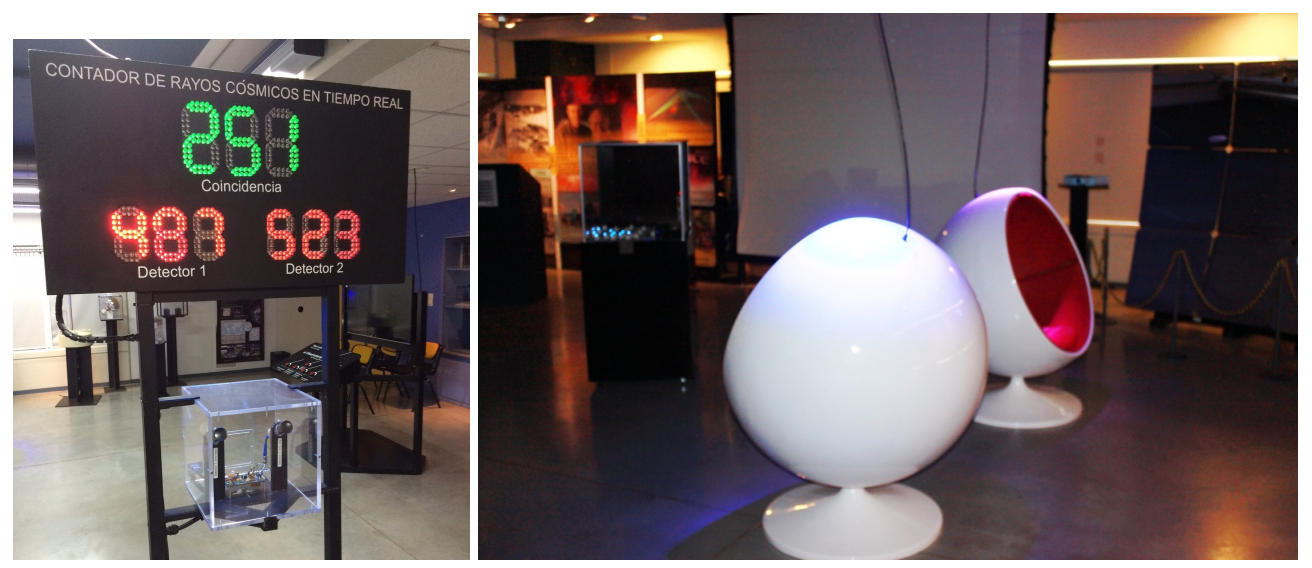

Figure 3: Left: A dual Geiger counter exhibit in the Visitor Center which registers single cosmic-ray muon counts and 2-fold coincidences. Right: Two "pods" in the renovated Visitor Center in which visitors can listen to an informative program about the Observatory.

\section{The 2016 Auger Science Fair}

The Observatory hosted its sixth biannual Science Fair in the Assembly Building on Nov. 1719, 2016, as shown in Fig. 4. Twenty-four student teams from all over Mendoza Province and beyond, with ages ranging from primary school through high school, presented research projects in the areas of natural science, exact science, and technology. More than 25 Auger collaborators and 
staff, of different nationalities, and a few invitees served as judges for the student projects. Prizes were awarded to the top teams in several categories in the closing ceremony on Nov. 19. The Nov. 2016 Science Fair owes its success to the Observatory staff, the collaborators who served as judges, the Municipality of Malargüe, the participating teachers and students, and the local organizers.

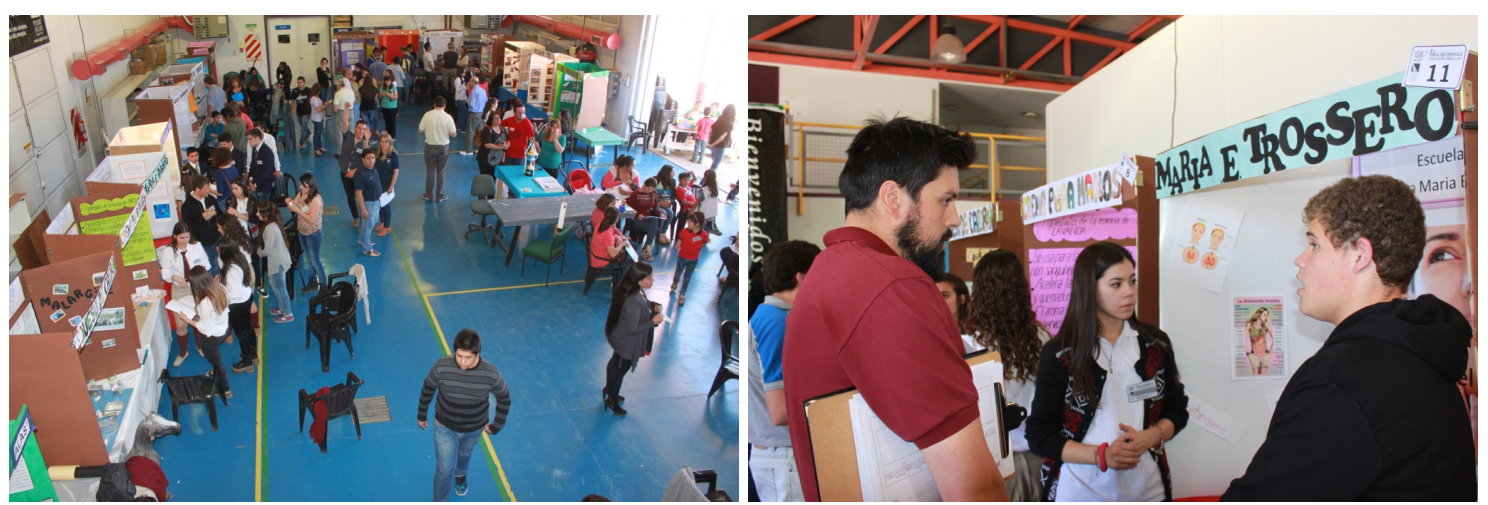

Figure 4: Left: The 2016 Science Fair in progress in the Assembly Building. Right: Auger staff member José Escalona judging one of the student projects at the Science Fair.

\section{Popularizing the AugerPrime upgrade and Auger science}

The collaboration was pleased to publish an article about the Observatory's planned upgrade in the June 2016 issue [3] of the CERN Courier, an international journal of high-energy physics whose readership spans the globe. The article, titled "AugerPrime looks to the highest energies", was featured on the cover of the issue (see Fig. 5) and explains the scientific motivation for the detector upgrades and highlights some of the improvements foreseen to enhance the experiment's performance. AugerPrime is expected to be completed in 2018 with little interruption to current data-taking operations.

The collaboration has also enhanced its online and social media presence in recent months. The Observatory's website (http://www.auger.org) now features accessible, "plain English" summaries of its journal publications, milestones reached in the AugerPrime upgrade project, and recognitions received by individual collaborators. Such news items are also shared on the Observatory's Twitter and Facebook pages more regularly.

The public data set [4] linked to the Auger website now contains over 45,000 Surface Detector events with energies between 0.1 and $49.7 \mathrm{EeV}$. The data set was accessed by over 1,500 users from Dec. 2015 to Dec. 2016, among which 70\% were new to the site. Collaborators at the RWTH Aachen University in Germany continue to make the data set more easily accessible to students and the general public through improvements to their user-friendly VISPA [5] analysis tools.

\section{Outreach activities around the world}

Many Auger Observatory collaborators engage in a wide range of outreach activities related to particle astrophysics at their institutions or in public venues. Recent activities of the Auger group from INFN in Lecce, Italy, are highlighted here. The group has developed a compact, portable cosmic-ray muon telescope [6], see Fig. 6, for use by high school students and teachers. The 

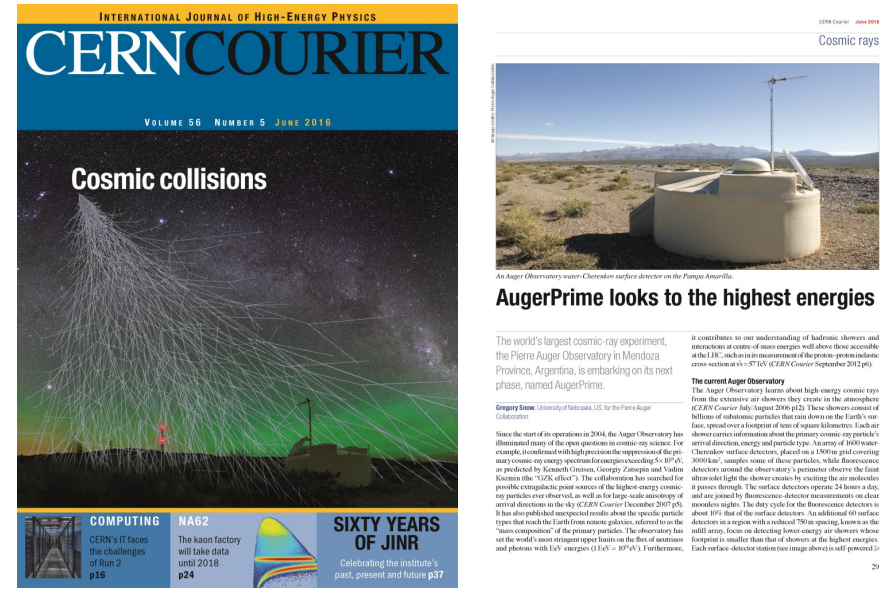

AugerPrime looks to the highest energies

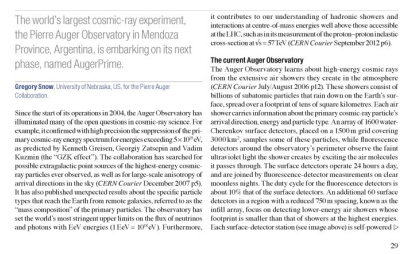

Figure 5: Issue cover (left) and first page (right) of the CERN Courier article about the AugerPrime upgrade.

detector consists of four $14.3 \times 14.3 \times 1.0 \mathrm{~cm}^{3}$ scintillator planes whose light is read out with embedded wavelength-shifting optical fibers that are attached to avalanche photo-diodes (APDs). The scintillators are interleaved with $2.0 \mathrm{~cm}$ thick lead plates so that a muon penetrating all four scintillators has a minimum energy of $150 \mathrm{MeV}$. The APDs and a GPS receiver are read out with a data-acquisition system which allows one to count 1-fold to 4-fold coincidences of the scintillator signals.
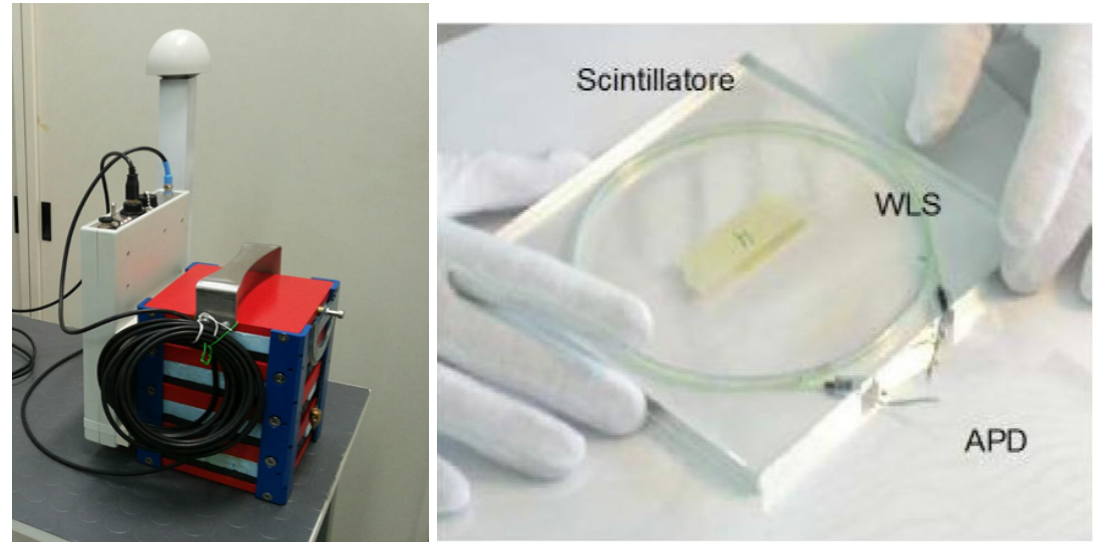

Figure 6: Left: The portable cosmic-ray telescope used by the Lecce group in Auger for outreach purposes, with its GPS receiver and data-acquisition system. Right: A scintillator plane with embedded wavelengthshifting fibers and avalanche photo-diode read out.

The detector has been used by high school students to measure the cosmic-ray muon flux at different altitudes up the Gran Sasso mountain, similar to the measurements made by Victor Hess during his well-known balloon flights. Fig. 7 shows other student measurements made both above ground and at the INFN laboratory in the Gran Sasso tunnel where the mountain's $1400 \mathrm{~m}$ rock overburden clearly shields the detector from cosmic-ray muons.

The Lecce group also participated in the Nov. 2016 International Cosmic Day (https://icd.desy.de), 

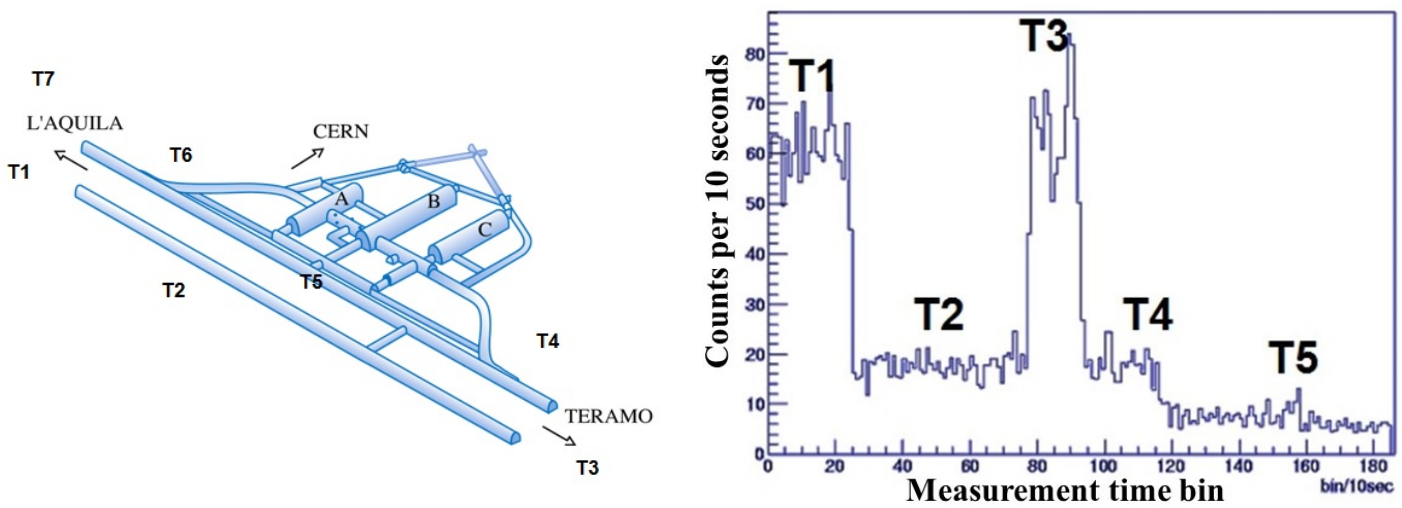

Figure 7: Left: The positions in the Gran Sasso tunnel where cosmic-ray muon flux measurements were made. Right: Muon rates at the different positions showing the effect of the mountain's overburden.

organized by DESY, Fermilab, and CERN. The group enlisted a large number of local university students in a day of presentations, laboratory tours, hands-on experiments, analyzing data, and sharing results with other participating students around the world by videoconference. The students in Lecce used the cosmic-ray telescope described above to measure the muon rates as a function of zenith angle by tilting the telescope from vertical to horizontal. The students prepared posters showing their results which became "proceedings contributions" to this distributed, international student conference on particle astrophysics.

\section{Conclusions}

The Pierre Auger Observatory continues to provide unique education and outreach opportunities which expose people of all ages to the excitement of astroparticle physics. Its Visitor Center and Science Fairs have great local impact near Malargüe, while collaborators around the world ensure that the Observatory's science and successes have international reach.

\section{References}

[1] C. Timmermans [Pierre Auger Collaboration], "Education, Outreach and Public Relations of the Pierre Auger Observatory," PoS ICRC 2015 (2016) 587; G. Snow [Pierre Auger Collaboration], "Education and Outreach Activities of the Pierre Auger Observatory," Proc. 33rd ICRC, Rio de Janeiro, Brazil, (2013) 116 [arXiv:1307.5059]; G. Snow [Pierre Auger Collaboration], "Education and Public Outreach of the Pierre Auger Observatory," Proc. 32nd ICRC, Beijing, China, 2 (2011) 190 [arXiv:1107.4806].

[2] See http://www.planetariomalargue.com.ar

[3] See http://cerncourier.com/cws/article/cern/65035

[4] See http://auger.colostate.edu/ED/

[5] See http://vispa.physik.rwth-aachen.de

[6] G. Chiarello, et al., "CORAM (COsmic RAy Mission): An outreach program one century after Pacini and Hess works", Nuclear Physics B (Proceedings Supplements) 239 (2013) 245-249. 